\title{
Zariski Closure of Subgroups of the Symplectic Group and Lyapunov Exponents of the Schrödinger Operator on the Strip
}

\author{
I.Ya. Goldsheid \\ Fakultät für Mathematik, Ruhr-Universität-Bochum, SFB-237, D-44780 Bochum, Germany
}

Received: 25 September 1994/in revised form: 1 February 1995

\begin{abstract}
We consider the Schrödinger equation with a random potential

$$
-y_{n+1}+\left(Q_{n}-E I\right) y_{n}-y_{n-1}=0,
$$

where $Q_{n}$ is a sequence of independent identically distributed random symmetric $m \times m$-matrices with real valued elements, $y_{n} \in \mathbb{R}^{m},-\infty<n<\infty, E$ is the real parameter, and $I$ is the identity matrix. We show that if the smallest Jordan algebra of matrices containing the support of the distribution of matrices $Q_{n}$ coincides with Jordan algebra of all (real-valued) symmetric matrices then for all but (maybe) a finite number of values of $E$ all the Lyapunov exponents of our Schrödinger equation are different (and thus the spectrum of the corresponding Schrödinger operator is pure point).
\end{abstract}

\section{General Remarks and Main Notations}

The study of spectral properties of random Schrödinger operators on a strip (the well-known Anderson model [A]) was initiated in [G] in 1980. At that time, the papers [GM1, GM2] did not yet exist and the case of potentials with singular distributions (singular potentials) couldn't be solved by means of the techniques used in $[\mathrm{G}]$. That is one of the reasons why localization has been proved there for operators with potentials having continuous density distribution. One more reason is worth mentioning. In [GMo, GMoP] localization has been understood for the first time and for the strictly one-dimensional case. The main goal of [G] was to go beyond the one-dimensional Anderson model and because of that the questions concerned with conditions of existence of any kind of densities were considered to be of much smaller importance compared with those related to the understanding of the mechanism of localization phenomena.

In [La1, La2] localization and exponential decay of the corresponding eigenfunctions has been established under similar conditions. A better understanding of the same phenomena was the main goal also for these works and the techniques used in [La1,La2] did not solve the case of singular potentials. 
After the appearance of [FS, FMSS] it became clear that, in order to prove localization, it is enough to establish certain regularity of the integrated density of states, to have some kind of weak dependence of potential values at different sites of the lattice, and positivity of Lyapunov exponents (the latter is equivalent to the exponential decay of Green's function). For the one-dimensional Anderson model with a singular potential, the corresponding program has been carried out in [CKM]. At that time the basic obstruction for the extension of the proof given in [CKM] to the case of the strip was the absence of any constructive conditions which would allow one to understand the structure of corresponding Lyapunov exponents. But as soon as this structure was described in [GM1], the preprint of [KLS] containing the proof of exponential localization on a strip for operators with singular potentials appeared. The same statement was independently announced in [GM3].

Today, as a result of this activity, in order to prove localization on a strip, one needs to establish that all the Lyapunov exponents of the product of corresponding independent symplectic matrices are different ([KLS]). In ([GM2]) this problem was reduced to the calculation of the Zariski closure of the group generated by the support of the distribution of these random matrices.

In the present paper, we provide a systematic approach for calculating Zariski closures of certain natural classes of groups of matrices generated by random matrix valued potentials. The description is complete for this class and is given in terms of properties of naturally associated Jordan algebras. The examples include in particular the so-called diagonal disorder (previously considered in [GM1, GM2]) and the new examples of the off-diagonal disorder acting in the "crossing" direction. Thus we indicate new classes of potentials for which exponential localization holds.

We do not give a proof of localization here. It follows directly from the results of this work about Lyapunov exponents and the corresponding theorems from [KLS]. The following problem naturally arises in our context and remains unsolved: to prove localization for the classes of systems where Lyapunov exponents have multiplicity at least two. We indicate the corresponding classes of potentials and it is clear that localization should hold because the smallest non-negative Lyapunov exponent is strictly positive also in this case. It is clear in advance that the corresponding eigenfunctions would also have multiplicity at least two.

Let us make several remarks about references. First of all, our list of them is far from being exhaustive and the interested reader should use [BL, CL] for further information. However, the papers [L2] and [KS] should be mentioned here. It is shown in these papers that if the randomness of the potential is strong enough (the so-called unpredictability condition) then the top Lyapunov exponent is strictly positive for almost all values of energies. On the one hand this condition is much less restrictive than the independence of potentials at different sites of the lattice. On the other hand the methods of [L2] and [KS] do not provide the complete description of all Lyapunov exponents whereas the methods of [GM2] do that for independent potentials. The methods of the three approaches ([L2, KS, and GM1, GM2]) are different and an interesting task is to combine these different techniques in order to understand the complete structure of Lyapunov exponents for stationary potentials.

We give now the list of main notations which will be used in the sequel. The corresponding definitions can be found in the text of the article:

$\mathscr{T}$-closed set of $m \times m$ symmetric matrices; it arises as the support of measure $\mu$. $G_{\mathscr{T}}$ - the group generated by matrices $A=\left(\begin{array}{cc}Q & -I \\ I & 0\end{array}\right)$, where $Q \in \mathscr{T}$. 
$G_{\tilde{T}, E}$-the group generated by matrices $A=\left(\begin{array}{cc}Q-E & -I \\ I & 0\end{array}\right)$, where $Q \in \mathscr{T}, E$ is a real parameter and we usually write $Q-E$ instead of $Q-E I$.

$\mathscr{J}_{\mathscr{T}}$-the Jordan algebra of symmetric matrices generated by the set $\mathscr{T}$.

$G_{\mathscr{Y}_{\mathcal{T}}}$-the group generated by matrices $A=\left(\begin{array}{cc}Q & -I \\ I & 0\end{array}\right)$, where $Q \in \mathscr{T}_{\mathscr{T}}$.

$\mathscr{R}_{\mathscr{T}}$-the generalized Jordan algebra of symmetric matrices generated by the set $\mathscr{T}$.

$\mathscr{R}_{\mathscr{T}, E}$-the generalized Jordan algebra of symmetric matrices generated by the set $\{Q-E: Q \in \mathscr{T}\}$.

$\mathscr{L}_{\mathscr{T}}$-the linear space of matrices generated by the set of products $b_{1} b_{2}$, where $b_{1}, b_{2} \in \mathscr{R}_{\mathscr{T}}$.

$Z c(G)$-Zariski closure of the group $G$.

$L G$-Lie algebra of the Lie group $G$.

$L Z c(G)$-Lie algebra of the Lie group $Z c(G)$.

$I-m \times m$ identity matrix.

$J=\left(\begin{array}{cc}0 & -I \\ I & 0\end{array}\right)$.

\section{Introduction and Formulation of Results}

Let $(\Omega, \mathscr{B}, P)$ be a probability space, where $\Omega=\{\omega\}$ is the space of elementary events, $\mathscr{B}$ is a $\sigma$-algebra of measurable subsets of $\Omega$, and $P$ is a probability measure on the measurable space $(\Omega, \mathscr{B})$.

Consider the Schrödinger equation with a random potential

$$
-y_{n+1}+\left(Q_{n}-E\right) y_{n}-y_{n-1}=0 \text {, }
$$

where $Q_{n}=Q_{n}(\omega)$ is a sequence of independent identically distributed (i.i.d.) random symmetric $m \times m$-matrices with real valued elements, $y_{n} \in \mathbb{R}^{m},-\infty<$ $n<\infty$. $E$ is the real parameter and $Q-E$ is just a short notation for $Q-E I$ with $I$ being the identity matrix. By $\mu$ we will denote the distribution of matrices $Q_{n}$ :

$$
\mu(\Gamma)=P\left\{Q_{n} \in \Gamma\right\},
$$

where $\Gamma$ is any Borel subset of the space of symmetric matrices.

By $\mathscr{T}$ we denote the support of the measure $\mu$ which by definition is the largest closed subset of the space of symmetric matrices such that the $\mu$-measure of every open neighborhood of every point of $\mathscr{T}$ is strictly positive.

Throughout this article we suppose that

$$
\int \log (\|Q\|+1) \mu(d Q)<\infty
$$

Equation (1) can be rewritten in the form

$$
\left(\begin{array}{c}
y_{n+1} \\
y_{n}
\end{array}\right)=\left(\begin{array}{cc}
Q_{n}-E & -I \\
I & 0
\end{array}\right)\left(\begin{array}{c}
y_{n} \\
y_{n-1}
\end{array}\right) \equiv A_{n}\left(\begin{array}{c}
y_{n} \\
y_{n-1}
\end{array}\right)
$$


and, thus, the asymptotic behavior of the solutions of Eq. (1) is determined by the asymptotic behavior of the product of matrices $A_{n}$,

$$
S(n):=A_{n} \cdots A_{1} \text {. }
$$

The aim of this paper is to give sufficient conditions under which all the Lyapunov exponents of Eq. (1) are distinct.

We recall now several definitions and results related to our situation. Consider the following Cartan decomposition of $S(n)$ :

$$
S(n)=U(n) D(n) V(n),
$$

where $U, V \in O(2 m)$, and $D(n)=\operatorname{diag}\left(d_{1}(n), \ldots, d_{2 m}(n)\right)$ with $d_{1} \geqq d_{2} \geqq \cdots \geqq$ $d_{2 m}>0$. The $k^{\text {th }}$ Lyapunov exponent $\gamma_{k}$ is defined by

$$
\gamma_{k}:=\lim _{n \rightarrow \infty} \frac{1}{n} \log d_{k}(n)
$$

Existence of the limits (3) for almost every $\omega$ and their independence of $\omega$ are guaranteed by the Subadditive Ergodic Theorem [Ki] and ergodicity of the underlying dynamical system (which in our case is just a consequence of i.i.d. property of the sequence of matrices $Q_{n}$ ). The meaning of values $\gamma_{k}$ is explained by Oseledets' multiplicative ergodic theorem (s.f. [O]; more recent versions of the proof can be found e.g. in [L1, GM2, R]). Namely, there exists a random basis $f_{1}(\omega), \ldots, f_{2 m}(\omega)$ in $\mathbb{R}^{2 m}$ such that

$$
\lim _{n \rightarrow \infty} \frac{1}{n} \log \left\|S(n) f_{k}\right\|=\gamma_{k}
$$

and for every vector $x \in \mathbb{R}^{2 m}$ there exists

$$
\lim _{n \rightarrow \infty} \frac{1}{n} \log \|S(n) x\|=\gamma(x)
$$

with $\gamma(x)=\gamma_{k}$ if and only if $x=a_{k} f_{k}+\cdots+a_{2 m} f_{2 m}$, where $\left|a_{k}\right|>0$.

We turn now to our special case. It is straightforward to check that matrices of the form

$$
A=\left(\begin{array}{cc}
Q-E & -I \\
I & 0
\end{array}\right)
$$

with $Q=Q^{*}$ satisfy the following equation:

$$
A^{*} J A=J \text {. }
$$

Here $J$ is given by

$$
J=\left(\begin{array}{cc}
0 & -I \\
I & 0
\end{array}\right) .
$$

The set of all real matrices $A$ satisfying Eq. (5) with our specifically chosen $J$ is by definition the symplectic group $S p(m, \mathbb{R})$. It follows from $(5)$ that

$$
d_{k}=d_{2 m-k+1}^{-1} \text {. }
$$

Clearly, $\gamma_{k}=-\gamma_{2 m-k+1}$, and

$$
\gamma_{1} \geqq \gamma_{2} \geqq \cdots \geqq \gamma_{m} \geqq 0 .
$$


We need one general result from [GM2] (it was first stated in [GM1]). The way we formulate it here is most suitable for our particular case of the symplectic group.

Theorem 1.1. Suppose that $A_{n}$ is a sequence of i.i.d. matrices belonging to $\operatorname{Sp}(m, \mathbb{R})$ and such that the Zariski closure of the group $G$ generated by the support of the distribution of $A_{n}$ coincides with $S p(m, \mathbb{R})$. Then all the Lyapunov exponents of the product $S_{n}=A_{n} \cdots A_{1}$ are different and the smallest non-negative one is strictly positive:

$$
\gamma_{1}>\gamma_{2}>\cdots>\gamma_{m}>0
$$

We recall that the Zariski closure of a subset $B \subset \mathbb{R}^{N}$ is the smallest algebraic variety which contains this set. In the sequel $Z c(B)$ will denote the Zariski closure of $B$.

It turns out that it is convenient to formulate our results in terms of the following definition.

Definition. A linear space of symmetric matrices $\mathscr{J}$ is a Jordan algebra if it has the following property:

$$
\text { if } a, b \in \mathscr{J} \text { then }\{a, b\}:=a b+b a \in \mathscr{J} \text {. }
$$

Denote by $\mathscr{J}_{\mathscr{T}}$ the Jordan algebra of symmetric matrices generated by the set $\mathscr{T}$, that is to say, the smallest Jordan algebra which contains $\mathscr{T}$.

We say that the spectrum of a symmetric matrix $Q$ is exceptional if at least one eigenvalue $\lambda$ of $Q$ is such that $-\lambda$ also belongs to the spectrum of $Q$ (let us note that if $\lambda=0$ belongs to the spectrum then the spectrum is exceptional).

Definition. We say that the real value $E_{0}$ is exceptional if the spectrum of the operators $Q-E_{0}$ is exceptional simultaneously (!) for all $Q \in \mathscr{T}$.

Remark. It is clear that the number of exceptional points cannot be larger than $\frac{1}{2} m(m+1)$ because this is the maximal possible number of exceptional points of one matrix. It is also clear that if the set $\mathscr{T}$ contains at least two matrices it is very "reasonable" then to expect that the set of exceptional points will be empty. Nonetheless, the exceptional finite set of $E$ 's mentioned above may really exist. In the last section we give an example of a distribution such that its support $\mathscr{T}$ is irreducible but $\gamma_{1}=\gamma_{2}$ for one particular exceptional value of $E$.

We are now prepared to formulate the main results of this paper.

Theorem 1.2. Suppose that the set $\mathscr{T}$ is such that the Jordan algebra $\mathscr{J}_{\mathscr{T}}$ coincides with the set of all $m \times m$ symmetric matrices, then for all but (maybe) a finite number of the exceptional values of the parameter $E$ the first $m$ Lyapunov exponents of the sequence of matrices $S(n)$ are different and the smallest non-negative one is strictly positive.

Let $G_{\mathscr{T}, E}$ be the group generated by matrices (4). It is clear that Theorem 1.2 is a consequence of Theorem 1.1 and the following result.

Theorem 1.3. If the set $\mathscr{T}$ is such that the Jordan algebra $\mathscr{J}_{\mathscr{T}}$ coincides with the set of all symmetric matrices, then for all but (maybe) a finite number of the 
exceptional values of the parameter $E$

$$
Z c\left(G_{\mathscr{T}, E}\right)=\operatorname{Sp}(m, \mathbb{R}) .
$$

In turn, Theorem 1.3 is a consequence of the two following results.

Theorem 1.4. If the set $\mathscr{T}$ is irreducible then for all but (maybe) a finite number of the exceptional values of $E$

$$
Z c\left(G_{\mathscr{T}, E}\right)=Z c\left(G_{\mathscr{J}_{\mathscr{T}}}\right)
$$

where $G_{\mathscr{Y}_{\mathscr{T}}}$ is the group generated by all matrices of the form $\left(\begin{array}{cc}Q & -I \\ I & 0\end{array}\right)$ with $Q \in \mathscr{J}_{\mathscr{T}}$.

Lemma 1.5. Suppose that the set $\mathscr{T}$ is irreducible and $\mathscr{J}_{\mathscr{T}}$ contains a projector of rank one. Then $\mathscr{Z}_{\mathscr{T}}$ is the set of all symmetric matrices and

$$
Z c\left(G_{\mathscr{Y}_{\mathscr{T}}}\right)=\operatorname{Sp}(m, \mathbb{R}) .
$$

Remark. The irreducibility of the set $\mathscr{T}$ means by definition that no nontrivial subspace of $\mathbb{R}^{m}$ is invariant with respect to the action of $Q$ on this subspace for all $Q \in \mathscr{T}$. In other words we suppose that there does not exist an orthogonal matrix $C$ such that simultaneously for all $Q \in \mathscr{T}$

$$
C^{*} Q C=\left(\begin{array}{cc}
Q^{\prime} & 0 \\
0 & Q^{\prime \prime}
\end{array}\right),
$$

with the sizes of blocks $Q^{\prime}$ and $Q^{\prime \prime}$ independent of $Q$. It is easy to see that if $\mathscr{T}$ is reducible then our system of equations (1) is equivalent to its two disjoint subsystems of exactly the same structure. To see this, one has just to make the following change of variables: $y_{n}^{\prime}=C y_{n}$. It is also clear that if $\mathscr{T}$ is reducible then (8) is false; in this case $Z_{c}\left(G_{\mathscr{T}, E}\right) \subset S p\left(m_{1}, \mathbb{R}\right) \times S p\left(m_{2}, \mathbb{R}\right)$, where $m_{1}$ and $m_{2}$ are the corresponding sizes of blocks $Q^{\prime}$ and $Q^{\prime \prime}$. So, the irreducibility condition is in a natural sense the necessary one.

All the new results formulated above contain the reference to exceptional points. However in many cases, the exceptional points do not exist. The corresponding classes of potentials have to be a bit richer of course. For applications, it suffices rather often to use the following result.

Theorem 1.6. If the set $\mathscr{T}$ is irreducible and there exist two operators $Q, \bar{Q} \in \mathscr{T}$ such that $Q-\bar{Q}$ is proportional to a projector of rank one, then $\mathscr{J}_{\mathscr{T}}$ contains all symmetric matrices and for all values of $E$ relation (8) holds.

\section{Calculation of the Zariski Closure of $G_{\mathscr{T}, E}$ and Proofs of Results}

In what follows, we denote by $L G$ the Lie algebra of a Lie group $G$.

The plan of this section is the following one. First, we fix an arbitrary value of the parameter $E$ and for this $E$ describe the Lie algebra of the Zariski closure of $G_{\mathscr{T}, E}$. This description is the main technical result of the paper and is formulated as Theorem 2.1 below. We show then that $G_{\mathscr{T}, E}$ is either a subgroup of $G_{0}$ (where by 
$G_{0}$ we denote the connected component of the identity of $\left.Z c\left(G_{\mathscr{T}, E}\right)\right)$ or belongs to the subgroup $G_{0} \cup J G_{0}$ which consists of two connected components of $Z c\left(G_{\mathscr{T}, E}\right)$. These results hold under the only condition that the set $\mathscr{T}$ is irreducible. After that, under the additional condition of existence of a rank one projector in $\mathscr{J}_{\mathscr{T}}$ we show that $L Z c\left(G_{\mathscr{T}, E}\right)=L S p(m, \mathbb{R})$ and thus $Z c\left(G_{\mathscr{T}, E}\right)=S p(m, \mathbb{R})$ for non-exceptional values of $E$. Altogether this constitutes the proofs of Theorem 1.4 and Lemma 1.5 and according to the explanations given above proves Theorems 1.2 and 1.3. Finally, we show that Theorem 1.6 also follows from Theorem 2.1.

2.1. Some Preliminary Calculations. First of all let us note that $Z c\left(G_{\overparen{T}, E}\right)$ is a Lie group. As far as $S p(m, \mathbb{R})$ is a connected group, to prove (8) it is enough to show that Lie algebras of the groups $Z c\left(G_{\mathscr{T}, E}\right)$ and $\operatorname{Sp}(m, \mathbb{R})$ coincide:

$$
\operatorname{LZc}\left(G_{\tilde{J}, E}\right)=\operatorname{LSp}(m, \mathbb{R}) .
$$

It is a well known and elementary fact that Lie algebra of $S p(m, \mathbb{R})$ consists of matrices of the form

$$
z=\left(\begin{array}{cc}
a & b_{1} \\
b_{2} & -a^{*}
\end{array}\right)
$$

where $a$ is an arbitrary $m \times m$-matrix, and $b_{1}, b_{2}$ are arbitrary symmetric $m \times m$ matrices: $b_{1}=b_{1}^{*}, b_{2}=b_{2}^{*}$. We have to show that for all but a finite number of values of $E$ all the matrices of this form belong to $L Z c\left(G_{\mathscr{T}, E}\right)$. In fact, we are going to do more than that. Namely, we describe the Lie algebra of $Z c\left(G_{\mathscr{T}, E}\right)$ for arbitrary $E$. For this description we don't need the properties like the existence of rank one projectors (which were mentioned in our theorems). These properties will only be used for descriptions of $L Z c\left(G_{\mathscr{T}, E}\right)$ and $Z c\left(G_{\mathscr{T}, E}\right)$ for "typical" values of $E$.

Let us start with a remark which uses a simple property of Zariski closure. Namely, if

$$
A_{1}=\left(\begin{array}{cc}
Q_{1}-E & -I \\
I & 0
\end{array}\right), A_{2}=\left(\begin{array}{cc}
Q_{2}-E & -I \\
I & 0
\end{array}\right) \in G_{\mathscr{T}, E},
$$

then

$$
\begin{aligned}
& \left(A_{1} A_{2}^{-1}\right)^{n}=\left(\begin{array}{cc}
I & n\left(Q_{1}-Q_{2}\right) \\
0 & I
\end{array}\right) \in G_{\mathscr{T}, E}, \\
& \left(A_{1}^{-1} A_{2}\right)^{n}=\left(\begin{array}{cc}
I & 0 \\
n\left(Q_{1}-Q_{2}\right) & I
\end{array}\right) \in G_{\mathscr{T}, E} .
\end{aligned}
$$

Evidently matrices of form (12) and (13) constitute subgroups of $G_{\mathscr{T}, E}$ and thus $Z c\left(G_{\mathscr{T}, E}\right)$ contains the Zariski closure of these subgroups. So, we have

$$
\left(\begin{array}{cc}
I & \alpha\left(Q_{1}-Q_{2}\right) \\
0 & I
\end{array}\right) \in Z c\left(G_{\mathscr{T}, E}\right), \quad\left(\begin{array}{cc}
I & 0 \\
\alpha\left(Q_{1}-Q_{2}\right) & I
\end{array}\right) \in Z c\left(G_{\mathscr{T}, E}\right),
$$

where $\alpha$ is an arbitrary real number. It follows from (14) that

$$
\left(\begin{array}{cc}
0 & \alpha\left(Q_{1}-Q_{2}\right) \\
0 & 0
\end{array}\right) \in \operatorname{LZc}\left(G_{\mathscr{T}, E}\right), \quad\left(\begin{array}{cc}
0 & 0 \\
\alpha\left(Q_{2}-Q_{1}\right) & 0
\end{array}\right) \in \operatorname{LZc}\left(G_{\mathscr{T}, E}\right) .
$$


2.2. Description of the Lie algebra $L Z c\left(G_{\mathscr{T}}\right)$. For a while, the fact that our group is the Zariski closure of $G_{\mathscr{T}, E}$ will not be used for the calculation of the Lie algebra $L Z c\left(G_{\mathscr{T}, E}\right)$. All that we need are properties (14) and (15), the irreducibility condition, and also the special form of generating matrices given by expression (4). We are also not going to use the dependence of our matrices on the parameter $E$. Let

$$
A=\left(\begin{array}{cc}
Q & -I \\
I & 0
\end{array}\right)
$$

where $Q \in \mathscr{T}$ and let $G_{\mathscr{T}}$ be the group generated by matrices (16).

Denote by $\mathscr{R}$ the set of symmetric $m \times m$-matrices which is defined as follows:

$$
\mathscr{R}=\left\{b:\left(\begin{array}{ll}
0 & b \\
0 & 0
\end{array}\right) \in \operatorname{LZc}\left(G_{\mathscr{T}}\right) \text { and } \quad\left(\begin{array}{ll}
0 & 0 \\
b & 0
\end{array}\right) \in \operatorname{LZc}\left(G_{\mathscr{T}}\right)\right\} .
$$

Let $\mathscr{L}$ be the set of $m \times m$-matrices such that

$$
\mathscr{L}=\left\{a:\left(\begin{array}{cc}
a & 0 \\
0 & -a^{*}
\end{array}\right) \in \operatorname{LZc}\left(G_{\mathscr{T}}\right) \quad \text { and } \quad\left(\begin{array}{cc}
a^{*} & 0 \\
0 & -a
\end{array}\right) \in \operatorname{LZc}\left(G_{\mathscr{T}}\right)\right\} .
$$

Evidently, $\mathscr{R}$ and $\mathscr{L}$ are linear spaces and it is clear from (15) that $\mathscr{R}$ is nontrivial.

Remark. The definitions of $\mathscr{R}$ and $\mathscr{L}$ are using the symmetric part of $\operatorname{LZc}\left(G_{\mathscr{T}}\right)$. Had we known that our Lie algebra is symmetric, that is that from $z \in \operatorname{LZc}\left(G_{\mathscr{T}}\right)$ it follows that $z^{*} \in L Z c\left(G_{\mathscr{T}}\right)$, we could have used simpler definitions like e.g.

$$
\mathscr{R}=\left\{b: \text { there exists } z \in L Z c\left(G_{\mathscr{T}}\right) \text { such that } z=\left(\begin{array}{ll}
0 & b \\
0 & 0
\end{array}\right)\right\} .
$$

We will see later that in fact $L Z c\left(G_{\mathscr{T}}\right)$ is symmetric. More precisely, we will prove that $J \in Z c\left(G_{\mathscr{T}}\right)$. The symmetry property is then a consequence from the following basic equation $z^{*} J+J z=0$ which is satisfied by the elements of our Lie algebra. This equation implies that $z^{*}=-J z J^{-1} \in L Z c\left(G_{\mathscr{T}}\right)$ if $z \in \operatorname{LZc}\left(G_{\mathscr{T}}\right)$.

We shall use several relations between linear spaces $\mathscr{L}$ and $\mathscr{R}$. These relations result immediately from the application of the basic Lie operation to matrices

$$
x=\left(\begin{array}{cc}
a & 0 \\
0 & -a^{*}
\end{array}\right), \quad y=\left(\begin{array}{cc}
0 & b \\
0 & 0
\end{array}\right), \quad z=\left(\begin{array}{cc}
0 & 0 \\
c & 0
\end{array}\right) .
$$

Namely, the direct calculation of $y z-z y$ and $x y-y x$ shows that

$$
\begin{array}{r}
\text { if } b, c \in \mathscr{R} \quad \text { then } \quad b c \in \mathscr{L}, \\
\text { if } a \in \mathscr{L}, b \in \mathscr{R} \quad \text { then } a b+b a^{*} \in \mathscr{R} .
\end{array}
$$

Now, from (20), (21) it follows that

$$
\text { if } b, c \in \mathscr{R} \text { then } b c b \in \mathscr{R} \text {. }
$$

Definition. We say that the linear space of $m \times m$ symmetric matrices $\mathscr{R}$ is a generalized Jordan algebra (GJA) if it satisfies property (22). 
Remark. Let us check that a Jordan algebra is also a GJA. Indeed, if $b, c \in \mathscr{J}$ then $\{\{c, b\} b\}=b^{2} c+c b^{2}+2 b c b \in \mathscr{J}$ and thus $b c b \in \mathscr{J}$.

Let $\mathscr{R}_{\mathscr{T}}$ be the smallest GJA which contains $\mathscr{T}$ and $\mathscr{L}_{\mathscr{T}}$ the smallest linear space of matrices containing all the products of the form $b c$ where $b, c \in \mathscr{R}_{\mathscr{T}}$.

Theorem 2.1. If the set $\mathscr{T}$ is irreducible then the Lie algebra $L Z_{c}\left(G_{\mathscr{T}}\right)$ consists of matrices given by (10) with arbitrary $a \in \mathscr{L}_{\mathscr{T}}$ and arbitrary $b_{1}, b_{2} \in \mathscr{R}_{\mathscr{T}}$.

Proof. The following general statement can be easily checked by means of a direct calculation. Suppose that $\tilde{\mathscr{R}}$ and $\tilde{\mathscr{L}}$ are linear spaces of matrices, $\tilde{\mathscr{R}}$ satisfies property (22), and $\tilde{\mathscr{L}}$ is generated by all the products of the form $b c$ where $b, c \in \tilde{\mathscr{R}}$. Then the set of matrices (10) with arbitrary $a \in \tilde{\mathscr{L}}$ and arbitrary $b_{1}, b_{2} \in \tilde{\mathscr{R}}$ is a Lie algebra.

In particular this is also true if $\tilde{\mathscr{R}}=\mathscr{R}_{\mathscr{T}}$ and $\tilde{\mathscr{L}}=\mathscr{L}_{\mathscr{T}}$. Let us denote this Lie algebra $L_{0}$. Now we go back to our special $\mathscr{R}$ and $\mathscr{L}$ and show that in our case $\mathscr{R}=\mathscr{R}_{\mathscr{T}}$ and thus $\operatorname{LZc}\left(G_{\mathscr{T}}\right)=L_{0}$.

We show first of all that $\mathscr{R} \supset \mathscr{R}_{\mathscr{T}}$. To do that we need several more properties of $\mathscr{R}$ and $\mathscr{L}$. It follows from (20) and (21) that

$$
\begin{aligned}
& \text { if } b \in \mathscr{R} \quad \text { then } \quad b^{2 n+1} \in \mathscr{R}, \quad n=1,2, \ldots, \\
& \text { if } b \in \mathscr{R} \quad \text { then } \quad b^{2 n} \in \mathscr{L}, \quad n=1,2, \ldots .
\end{aligned}
$$

Next, the set $\mathscr{T}$ contributes to the enlargement of $\mathscr{R}$ (and thus also of $\mathscr{L}$ ) in the following way. If $A$ and $x$ are given by (16) and (19) then

$$
\begin{aligned}
A x A^{-1} & =\left(\begin{array}{cc}
-a^{*} & Q a+a^{*} Q \\
0 & a
\end{array}\right) \in \operatorname{LZc}\left(G_{\mathscr{T}}\right), \\
A^{-1} x^{*} A & =\left(\begin{array}{cc}
-a & 0 \\
-Q a-a^{*} Q & a^{*}
\end{array}\right) \in \operatorname{LZc}\left(G_{\mathscr{T}}\right),
\end{aligned}
$$

and thus

$$
\text { if } a \in \mathscr{L}, Q \in \mathscr{T} \quad \text { then } \quad d:=Q a+a^{*} Q \in \mathscr{R} .
$$

Combining (21) and (26) we obtain

$$
\text { if } a \in \mathscr{L}, Q \in \mathscr{T} \quad \text { then } a d+d a^{*}=a Q a+a^{*} Q a^{*}+a a^{*} Q+Q a a^{*} \in \mathscr{R} \text {. }
$$

Lemma 2.2. If $\mathscr{T}$ is irreducible then $I \in \mathscr{L}$.

Proof. Consider $b \in \mathscr{R}, b \neq 0$. Then

$$
b^{2}=\sum_{i=1}^{k} \lambda_{l} u_{i},
$$

where all $\lambda_{l}$ 's are different eigenvalues of $b^{2}$ and $u_{l}$ are corresponding orthogonal projectors. If $\lambda_{s} \neq 0$ then evidently

$$
u_{s}=\left(b^{2} / \lambda_{s}\right) \prod_{\lambda_{i} \neq \lambda_{s}, \lambda_{l} \neq 0}\left(\lambda_{s}-\lambda_{l}\right)^{-1}\left(b^{2}-\lambda_{l}\right),
$$


and $u_{s} \in \mathscr{L}$ because of (24). Consider now the following projector:

$$
u=\sum_{i: \lambda_{i} \neq 0} u_{l} \in \mathscr{L}
$$

Obviously, $\operatorname{Rank} u=\operatorname{Rank} b=\operatorname{Rank} b^{2}$. Thus $u=I$ iff $\operatorname{Rank} b^{2}=m$. In this case, the proof is finished.

Suppose now that Rank $b^{2}<m$. We will then use the projector $u$ to construct another projector $v \in \mathscr{L}$ with the property Rank $v>\operatorname{Rank} u$. This would obviously mean that the maximal rank of projectors belonging to $\mathscr{L}$ is $m$ and would complete the proof of our lemma.

To construct $v$, let us substitute $a=u$ into (27). We obtain then

$$
2 u Q u+u Q+Q u \in \mathscr{R}
$$

and thus

$$
u Q u \in \mathscr{R}
$$

and

$$
h:=u Q+Q u-2 u Q u \in \mathscr{R} .
$$

Let $\pi$ denote the kernel of $u$ and $\pi^{\perp}$ be the subspace onto which $u$ is projecting. It is easy to check that $h^{2} \in \mathscr{L}$ and preserves $\pi$ (and of course also $\pi^{\perp}$ ).

If $\phi \in \pi$, then $h \phi=u Q \phi$ and $h^{2} \phi=(I-u) Q u Q \phi$. Using the relation $u \phi=0$ we obtain

$$
\left(h^{2} \phi, \phi\right)=(Q u Q \phi,(I-u) \phi)=(u Q \phi, Q \phi)=\|u Q \phi\|^{2} .
$$

We choose now $\phi$ from $\pi$ and $Q \in \mathscr{T}$ so that $Q \phi \notin \pi$. The latter is possible because $\mathscr{T}$ is irreducible. Then $\left(h^{2} \phi, \phi\right)>0$. It is evident that $\operatorname{Rank}\left(h^{2}+u\right)>\operatorname{Rank} u$ and $h^{2}+u \in \mathscr{L}$. Now we obtain $v$ from $h^{2}+u$ in the same way we have already constructed $u$ from $b^{2}$. Then $\operatorname{Rank} v=\operatorname{Rank}\left(h^{2}+u\right)$. The lemma is proven.

Corollary. Substituting $I \in \mathscr{L}$ into (26) we obtain that

$$
\text { if } Q \in \mathscr{T} \text { then } Q \in \mathscr{R} \text {. }
$$

It is now a trivial consequence of (22) and (36) that $\mathscr{R} \supset \mathscr{R}_{\mathscr{T}}$ and thus also $\mathscr{L} \supset$ $\mathscr{L}_{\mathscr{T}}$ and

$$
\operatorname{LZc}\left(G_{\mathscr{T}}\right) \supset L_{0} .
$$

We shall prove now that in fact the following equality holds:

$$
L Z c\left(G_{\mathscr{T}}\right)=L_{0}
$$

To do that we use the fact that we are dealing with a Zariski closure.

Lemma 2.3. The matrix $J$ given by (6) belongs to $Z c\left(G_{\mathscr{T}}\right)$.

Proof. If $A$ is given by (16), then it follows from (36) that $\left(\begin{array}{cc}0 & -Q \\ 0 & 0\end{array}\right) \in L_{0}$ and therefore

$$
\exp \left(\begin{array}{cc}
0 & -Q \\
0 & 0
\end{array}\right)=\left(\begin{array}{cc}
I & -Q \\
0 & I
\end{array}\right) \in G_{0}
$$


(We recall that $G_{0}$ is by definition the connected component of the identity of $Z c\left(G_{\mathscr{T}}\right)$.) But $A \in Z c\left(G_{\mathscr{T}}\right)$ by definition and thus

$$
\left(\begin{array}{cc}
I & -Q \\
0 & I
\end{array}\right) A=\left(\begin{array}{cc}
0 & -I \\
I & 0
\end{array}\right) \in Z c\left(G_{\mathscr{T}}\right) .
$$

Corollary. $L Z\left(G_{\overparen{T}}\right)$ is symmetric. For proof, see the remark at the beginning of Sect. 2.2.

Lemma 2.4. The Lie algebra $L_{0}$ is algebraic (that is $L_{0}$ is a Lie algebra of some algebraic group).

Proof. Lemma 2.2 tells us that $x=\left(\begin{array}{cc}I & 0 \\ 0 & -I\end{array}\right) \in L_{0}$ and from (36) we know that $y=\left(\begin{array}{ll}0 & Q \\ 0 & 0\end{array}\right) \in L_{0}$. Evidently then $x y-y x=2 y \in L_{0}$. Thus, it is clear that the commutant of $L_{0}$ contains all the matrices $y$ with $Q \in \mathscr{T}$. This in turn means that the commutant of $L_{0}$ coincides with $L_{0}$ because $L_{0}$ is in fact generated as a Lie algebra by matrices $y, y^{*}$ with $Q \in \mathscr{T}$ (we use here the fact that $L_{0}$ is symmetric). Consider now the standard complexification $\tilde{L}_{0}$ of $L_{0}$ :

$$
\tilde{L}_{0}:=\left\{z_{1}+i z_{2}: z_{1}, z_{2} \in L_{0}, i^{2}=-1\right\} .
$$

Clearly, the commutant of $\tilde{L}_{0}$ is again $\tilde{L}_{0}$. According to a well known theorem of Chevalley (see e.g. [OV]) any complex Lie group is algebraic if the Lie algebra of this group coincides with its commutant. Thus the group generated by $\tilde{L}_{0}$ is algebraic. But then the real subgroup of the latter complex Lie group is algebraic. This proves that $L_{0}$ is algebraic, because it is the Lie algebra of this subgroup.

Let $M$ be the group generated by $L_{0}$. Consider, next, $Z c(M)$. Evidently, $Z c(M) \subset Z c\left(G_{\mathscr{T}}\right)$.

Theorem 2.5. Suppose that $\mathscr{T}$ is irreducible. Then

1. $J \in L_{0}$ implies $Z c\left(G_{\mathscr{T}}\right)=Z c(M)$ and $G_{\mathscr{T}} \subset G_{0}$;

2. $J \notin L_{0}$ implies $Z c\left(G_{\mathscr{T}}\right)=Z c(M) \cup J Z c(M)$ and $G_{\mathscr{T}} \subset G_{0} \cup J G_{0}$, where $J$ is the matrix given by (6).

Proof. Suppose first that $J \in L_{0}$. Then for every real $t$,

$$
\exp (t J)=\left(\begin{array}{cc}
\cos t I & -\sin t I \\
\sin t I & \cos t I
\end{array}\right) \in M
$$

In particular,

$$
\exp \left(\frac{\pi}{2} J\right)=J \in M
$$

but then using (38) we obtain that

$$
A=\left(\begin{array}{cc}
I & Q \\
0 & I
\end{array}\right)\left(\begin{array}{cc}
0 & -I \\
I & 0
\end{array}\right)=\left(\begin{array}{cc}
Q & -I \\
I & 0
\end{array}\right) \in M,
$$

and hence $M \supset G_{\mathscr{T}}$ and thus $Z c(M)=Z c\left(G_{\mathscr{T}}\right)$. 
Suppose now that $J \notin L_{0}$. As far as $L_{0}$ is symmetric (by construction) so is also $M$ and $Z c(M)$, that is if $g \in Z c(M)$, then $g^{*} \in Z c(M)$. Next, it follows from (5) that if $g \in Z c(M)$, then $J_{g}^{-1} J^{-1}=g^{*}$ and thus $J^{-1} J^{-1} \in Z c(M)$. Hence $J Z c(M) J^{-1}=Z c(M)$. Therefore, the union $Z c(M) \cup J Z c(M)$ is a group and moreover this group is algebraic. By Lemma $2.3 J \in Z c\left(G_{\mathscr{T}}\right)$ and the same calculation shows that in this case $A \in J M$. Theorem 2.5 is proven.

Finally, from Theorem 2.5 it follows that $L Z c\left(G_{\mathscr{T}}\right)=L Z c(M)=L_{0}$ which proves (37) and thus also finishes the proof of Theorem 2.1.

\subsection{Proofs of Theorem 1.4, Lemma 1.5, and Theorem 1.6}

Proof of Theorem 1.4. As explained in Theorem 2.1 and Lemma 2.4 the generalized Jordan algebra $\mathscr{R}_{\mathscr{T}}$ is responsible for the description of the Lie algebra $\operatorname{LCc}\left(G_{\mathscr{T}}\right)$. It is evident that if $I \in \mathscr{R}_{\mathscr{T}}$, then the generalized Jordan algebra is in fact a Jordan algebra: $\mathscr{R}_{\mathscr{T}}=\mathscr{J}_{\mathscr{T}}$. Thus, Theorem 1.4 is a consequence of the following simple observation: for all but (maybe) exceptional values of $E$,

$$
\mathscr{R}_{\mathscr{T}, E}=\mathscr{J}_{\mathscr{T}},
$$

which in turn follows from the fact that $I \in \mathscr{R}_{\mathscr{T}, E}$ for all but (maybe) exceptional values of $E$. We prove now the last statement. Let us fix $E$ which is not exceptional. Then there exists a matrix $Q \in \mathscr{T}$ such that the spectrum $h:=Q-E I$ is not exceptional. As usual, we can write

$$
h=\sum_{l=1}^{k} \lambda_{i} u_{i}
$$

where $\lambda_{l}$ 's are different eigenvalues of $h$ ordered so that $\left|\lambda_{1}\right|>\cdots>\left|\lambda_{k}\right|>0$ and $u_{i}$ are corresponding orthogonal projectors. The strict inequalities do have place because the spectrum of $h$ is not exceptional. As we know from (23) if $h \in \mathscr{R}_{\mathscr{T}}$ then $h^{2 n+1} \in \mathscr{R}_{\mathscr{T}}$, where $n=1,2, \ldots$ Evidently

$$
\lim _{n \rightarrow \infty} \lambda_{1}^{-2 n-1} h^{2 n+1}=u_{1} \in \mathscr{R}_{\mathscr{T}, E}
$$

Then of course

$$
\lim _{n \rightarrow \infty} \lambda_{2}^{-2 n-1}\left(h-\lambda_{1} u_{1}\right)^{2 n+1}=u_{2} \in \mathscr{R}_{\mathscr{T}, E}
$$

and similarly

$$
\lim _{n \rightarrow \infty} \lambda_{r}^{-2 n-1}\left(h-\sum_{i=1}^{r-1} \lambda_{l} u_{i}\right)^{2 n+1}=u_{r} \in \mathscr{R}_{\mathscr{T}, E} .
$$

Evidently $\sum_{i=1}^{k} u_{i}=I \in \mathscr{R}_{\mathscr{T}, E}$ and thus $\mathscr{R}_{\mathscr{T}, E}=\mathscr{J}_{\mathscr{T}}$ for all non-exceptional values of $E$.

Proof of Lemma 1.5. The fact that $J_{\mathscr{T}}$ contains all symmetric matrices is just a general property of Jordan algebras. We give the proof of this result in the Appendix (Lemma A3). But then the group $G_{\mathscr{F}_{T}}$ is generated by matrices (16) with arbitrary symmetric $Q$. This in turn means that the corresponding set $\mathscr{R}=\mathscr{J}_{\mathscr{T}}$. Hence, the corresponding $\mathscr{L}$ is the set of all $m \times m$ matrices and $L Z c\left(G_{\mathscr{F}_{\mathscr{T}}}\right)=L S p(m, \mathbb{R})$ and $Z c\left(G_{\mathscr{I}_{\mathscr{T}}}\right)=S p(m, \mathbb{R})$ 
Proof of Theorem 1.6. It follows from the conditions of our theorem that $\mathscr{J}_{\mathscr{T}, E}$ contains a one-dimensional projector for every real $E . \mathscr{J}_{\mathscr{T}, E}$ is also irreducible for every real $E$. Thus by Lemma A4 (see Appendix) $\mathscr{J}_{\mathscr{T}, E}$ coincides with all symmetric matrices and hence (8) holds for every $E$.

\section{Some Applications of Theorem 1.6}

We will show now that Theorem 1.6 provides the existence of different Lyapunov exponents in particular for the classical case of Jacobi matrices. The following two lemmas are very useful for that. Denote by $\delta_{l}$ the $m \times m$ matrix which has the only nonzero element $\left(\delta_{l}\right)_{i i}=1$.

Lemma 3.1. Suppose that $\mathscr{T}$ consist of Jacobi matrices, i.e. if $Q \in \mathscr{T}$ and $q_{\imath j}$ are the matrix elements of $Q$, then

$$
\begin{aligned}
& q_{i j}=-1, \quad \text { if } \quad|i-j|=1, \\
& q_{l j}=0, \quad \text { if } \quad|i-j| \geqq 2 .
\end{aligned}
$$

Suppose also that one of two following conditions is satisfied:

a) There exist two matrices $Q_{1}, Q_{2} \in \mathscr{T}$ such that $Q_{1}-Q_{2}=\alpha \delta_{1}$, where the real number $\alpha \neq 0$.

b) There exists $i,(1 \leqq i<m)$ and matrices $Q_{1}, Q_{2}, Q_{3}, Q_{4} \in \mathscr{T}$ such that

$$
\begin{aligned}
& Q_{1}-Q_{2}=\alpha_{l} \delta_{l}, \\
& Q_{3}-Q_{4}=\alpha_{l+1} \delta_{l+1} .
\end{aligned}
$$

Then $\mathscr{T}$ is irreducible, and thus $Z c\left(G_{\mathscr{T}}\right)=S p(m, \mathbb{R})$ and inequalities (7) hold.

Proof. We need only to establish that $\mathscr{T}$ is irreducible. We consider only the case b); in the case a) the proof is even simpler. Suppose that the statement of our lemma is not true. Consider then a nontrivial subspace $F$ (with $\operatorname{dim} F<m$ ), $F \subset \mathbb{R}^{m}$ which is a common invariant subspace of the matrices $Q \in \mathscr{T}$. As far as $Q=Q^{*}$ the orthogonal complement $F^{\perp}$ has the same property: $Q\left(F^{\perp}\right) \subset F^{\perp}$ for every $Q \in \mathscr{T}$. Thus from b) we have in particular $\delta_{l}(F) \subset F$ and $\delta_{i+1}(F) \subset F$. Obviously, vectors $e_{i}$ and $e_{i+1}$ of the standard basis of $\mathbb{R}^{m}$ are the eigenvectors of operators $\delta_{l}$ and $\delta_{l+1}$ with eigenvalues $\alpha_{i}$ and $\alpha_{i+1}$ correspondingly. The eigenvalue $\alpha_{i}$ has multiplicity one and therefore either $e_{l} \in F$ or $e_{i} \in F^{\perp}$. The same applies to $e_{i+1}$. Without loss of generality we can suppose that $e_{i} \in F$. The Jacobi structure of our matrices gives then

$$
Q e_{i}=e_{i-1}+q_{l i} e_{l}+e_{i+1} \in F .
$$

Evidently, $Q e_{i}$ and $e_{i+1}$ are not orthogonal and thus $e_{i+1} \in F$. But then $e_{l-1}=$ $Q e_{i}-q_{i i} e_{i}-e_{i+1} \in F$. Applying $Q$ to $e_{i-1}$ and $e_{i+1}$, we see that $e_{i-2}$ and $e_{i+2} \in F$ and so on. This procedure implies $F=\mathbb{R}^{m}$ and proves the lemma.

The same result is true for multidimensional Jacobi matrices. By definition this means that $Q=\left(q_{i j}\right)$ with $i$ and $j$ being multi-indices, $i=\left(i_{1}, i_{2}, \ldots, i_{k}\right), 1 \leqq$ $i_{s} \leqq m_{s}(s=1,2, \ldots, k)$ and $|i-j|=\sum_{s}\left|i_{s}-j_{s}\right|$. The Jacobi property means that 
$q_{i j}=-1$ if $|i-j|=1$ and $q_{i j}=0$ otherwise. Here are the conditions analogous to a) and b):

$\left.\mathrm{a}^{\prime}\right)$ Denote by $M_{s}:=\left\{i: i_{s}=m_{s}\right\}$. Let $s$ be fixed. We suppose that for any $i \in M_{s}$ there exist two matrices $Q^{\prime}(i), Q^{\prime \prime}(i) \in \mathscr{T}$ such that

$$
Q^{\prime}(i)-Q^{\prime \prime}(i)=\alpha_{i} \delta_{i} \quad \text { where } \quad \alpha_{l} \neq 0
$$

$\left.\mathrm{b}^{\prime}\right)$ Denote by $M_{s, r}=\left\{i: i_{s}=r\right\}$, where $s, r$ are fixed and $1<r<m_{s}$. We suppose that property (39) holds for all $i \in M_{s, r}$ and for any $i \in M_{s, r+1}$.

Lemma 3.2. If $\mathscr{T}$ is a set of multidimensional Jacobi matrices with properties $\left.\mathrm{a}^{\prime}\right)$ or $\left.\mathrm{b}^{\prime}\right)$, then $\mathscr{T}$ is an irreducible set of matrices and thus $Z c\left(G_{\mathscr{T}}\right)=S p(m, \mathbb{R})$, where $m:=m_{1} \cdot m_{2} \cdot \cdots \cdot m_{s}$.

The proof of Lemma 3.2 is similar to the one of Lemma 3.1, so we omit it.

Remarks. 1) In condition $\mathrm{a}^{\prime}$ ), we can replace $M_{s}$ by $\bar{M}_{s}:=\left\{i: i_{s}=1\right\}$. Evidently this does not change anything.

2) The condition $q_{i j}=1$ if $|i-j|=1$ for the case of Jacobi matrices can be replaced by the following one: "for any pair $i, j$ there exists $Q \in \mathscr{T}$ with $q_{l j} \neq 0$." In this case the result will be the same.

Finally, we will show that Theorem 7.3 from [GM2] is a consequence of Theorem 1.6. Let $\mathscr{I}$ be a finite set of indices.

\section{Theorem 3.3 ([GM2], Theorem 7.3). Suppose that}

(i) for every $i \in \mathscr{I}$ there are $Q^{\prime}(i), Q^{\prime \prime}(i) \in \mathscr{T}$ such that (39) holds.

(ii) for every pair of indices $i, j$ there exists a sequence of matrices $Q_{0}, Q_{1}, \ldots$, $Q_{s} \in \mathscr{T}$ and a sequence of indices $i_{1}, \ldots, i_{s} \in \mathscr{I}$ such that the product $q_{u_{1}}^{(0)} q_{i_{1} i_{2}}^{(1)}$ $\ldots q_{i_{s J}}^{(s)} \neq 0$. Here $q_{l j}^{(k)}$ are the matrix elements of $Q_{k}$. Then the inequalities (7) hold for every $E$.

Proof. All that we need is to check that the set $\mathscr{T}$ is irreducible or, equivalently, the algebra generated by $\mathscr{T}$ is irreducible. Suppose that on the contrary some nontrivial subspace $F \subset \mathbb{R}^{m}$ is preserved by all $Q \in \mathscr{T}$. Pick then a vector $x \in F$ and $i \in \mathscr{I}$ such that the scalar product $\left(x, e_{i}\right) \neq 0$ (here $e_{i}$ denotes the vector of a standard basis of $\mathbb{R}^{m}$ ). Then $\delta_{i} x=c e_{l}, c \neq 0$ and thus $e_{i} \in F$. Next, consider a matrix $Q \in \mathscr{T}$ such that its matrix element $q_{r l}=q_{l r} \neq 0$ for some $r, r \neq i$. Then $\delta_{r} Q e_{i}=q_{r l} e_{r}$ and hence $e_{r} \in F$. The condition (ii) allows to continue this process and to show that all $e_{j} \in F$ and thus $F=\mathbb{R}^{m}$.

\section{Examples of Equations with Lyapunov Exponents of Multiplicity Two}

We would like to discuss now two examples of the Schrödinger equation with off-diagonal disorder. 
Example 1. Consider Eq. (1) with $E=0, m=2$ and matrices $Q$ having the form

$$
Q=\left(\begin{array}{cc}
a & b \\
b & -a
\end{array}\right)
$$

It is easy to see that the set of all these matrices is a generalized Jordan algebra which we denote by $\mathscr{R}$. This set is not a Jordan algebra and this is exactly the case when $E=0$ is the only exceptional value of parameter $E$ (see the corresponding definition given in Sect. 1). The interesting case is the one when the set $\mathscr{T}$ contains at least two matrices. We suppose that the set $\mathscr{T}$ is irreducible and then at least two matrices from $\mathscr{T}$ are not proportional to each other. Thus

$$
\mathscr{R}=\mathscr{R}_{\mathscr{T}}
$$

For further considerations it is convenient to introduce the following three matrices:

$$
I_{1}=\left(\begin{array}{cc}
1 & 0 \\
0 & -1
\end{array}\right), \quad I_{2}=\left(\begin{array}{ll}
0 & 1 \\
1 & 0
\end{array}\right), \quad I_{3}=\left(\begin{array}{cc}
0 & 1 \\
-1 & 0
\end{array}\right) .
$$

Let us describe now the Lie algebra of the group $G_{\mathscr{T}}$. It follows from Theorem 2.1 that the Lie algebra of $L Z c\left(G_{\mathscr{T}}\right)$ consists of matrices given by

$$
z=\left(\begin{array}{cc}
a I+b I_{3} & c I_{1}+d I_{2} \\
\tilde{c} I_{1}+\tilde{d} I_{2} & -a I+b I_{3}
\end{array}\right) .
$$

It is a matter of elementary calculation to check that if $\tilde{J}=\left(\begin{array}{cc}0 & I_{3} \\ I_{3} & 0\end{array}\right)$ then

$$
z^{*} \tilde{J}+\tilde{J}_{z}=0 \text { for every } \quad z \in \operatorname{LZc}\left(G_{\mathscr{T}}\right) .
$$

The last relation is equivalent to the following equation:

$$
A^{*} \tilde{J} A=\tilde{J},
$$

which is satisfied by all matrices $A \in G_{0}$, where $G_{0}$ is the connected component of the identity of $Z c\left(G_{\mathscr{T}}\right)$. Now, the Lyapunov exponents have multiplicity two in our example. To check this statement, one can use the evident fact that the diagonal matrices from $L Z c\left(G_{\mathscr{T}}\right)$ are proportional to $\left(\begin{array}{cc}I & 0 \\ 0 & -I\end{array}\right)$ and thus the diagonal part of the Cartan decomposition equals $\left(\begin{array}{cc}d I & 0 \\ 0 & d^{-1} I\end{array}\right)$, where $d>0$. Hence, it follows from (3) that $\gamma_{1}=\gamma_{2}=-\gamma_{1}=-\gamma_{2}$.

Let us also indicate a simpler way to understand this fact. If $y_{n}$ is a sequence of two-dimensional vectors satisfying the equation

$$
-y_{n+1}+Q_{n} y_{n}-y_{n-1}=0 \text {, }
$$

where $Q_{n}$ are given by (40), then the sequence of vectors $z_{n}=(-1)^{n} I_{3} y_{n}$ also satisfies this equation. In particular this means that if solution $y_{n}$ of (43) is exponentially decaying at infinity then so does $z_{n}$, and it is evident that these solutions are linearly independent and $\left\|y_{n}\right\|=\left\|z_{n}\right\|$. 
Example 2. We keep notations used in Example 1. Consider now Eq. (1) with arbitrary $E, m=4$ and matrices $Q$ having the form

$$
Q=\left(\begin{array}{cc}
a I & b I+c I_{3} \\
b I-c I_{3} & d I
\end{array}\right)
$$

It is easy to check that if parameters $a, b, c, d$ are arbitrary real numbers, then the set of all matrices given by (44) is a Jordan algebra $\mathscr{J}$. It is not hard to show that every Jordan algebra of $4 \times 4$ symmetric matrices which is irreducible and does not contain any projector of rank one is equivalent to $\mathscr{J}$. According to Theorems $2.5,1.4$ the group $G_{\mathscr{T}, E}$ belongs to the connected component of the identity of $G_{0} \in Z c\left(G_{\mathscr{T}}\right)$ for all but a finite number of values of $E$. The group $G_{0}$ is generated by the algebra $L Z c\left(G_{\mathscr{J}}\right)$ (Theorem 2.1). It is not hard to describe $Z c\left(G_{\mathscr{T}}\right)$ by means of algebraic equations. To do that, let us introduce the following matrix $K:=\left(\begin{array}{cc}I_{3} & 0 \\ 0 & I_{3}\end{array}\right)$. A direct calculation shows that $K Q=Q K$. It is now easy to see that if $A$ is given by (4) with $Q$ given by (44) then $A$ is commuting with $\tilde{K}:=\left(\begin{array}{cc}K & 0 \\ 0 & K\end{array}\right)$ and thus all the elements of our group also satisfy the equation

$$
A \tilde{K}=\tilde{K} A \text {. }
$$

Thus, our Zariski closure is the set of non-degenerate matrices satisfying Eqs. (5) and (45). lowing:

Finally the structure of Lyapunov exponents of Eq. (1) in our case is the fol-

$$
\gamma_{1}=\gamma_{2}>\gamma_{3}=\gamma_{4}>0
$$

This can be deduced from the general results about Lyapunov exponents and the structure of the Lie algebra of our group in the same way as it has been done in the previous example. It is again useful to observe (analogously to what we have done in the previous example) that if $y_{n}$ is a sequence of four-dimensional vectors satisfying Eq. (1), where $Q$ 's are given by (44) then the sequence of vectors $z_{n}=K y_{n}$ also satisfies this equation. Thus, if a solution with negative Lyapunov exponent is given then we are able to indicate another solution which is linearly independent of the given one and has the same Lyapunov exponent. This proves that the smallest negative Lyapunov exponent has multiplicity at least two. To prove that it is equal to two, it is again necessary to use the general description of Lyapunov exponents from [GM1, GM2].

\section{Appendix: Several Remarks about Jordan Algebras of Symmetric Matrices}

All the Jordan algebras (JA) and generalised Jordan algebras (GJA) considered below are subalgebras of JA of $m \times m$ symmetric matrices. We need several simple results about these algebras. One could certainly find them in [BK]. However, we prove these results here which we hope simplifies reading this article for those who are not interested in general theory of JA.

Let $\mathscr{J}$ be a JA, $\mathscr{F} \subset \mathscr{J}$ be a set of all projectors of minimal rank belonging to $\mathscr{J}$, and $\mathscr{J}_{0}$ be the linear subspace of $\mathscr{J}$ generated by $\mathscr{F}$. By $s$ we denote the minimal rank of projectors belonging to $\mathscr{J}$. 
Remark. A priori $\mathscr{J}_{0}$ is not an algebra.

Lemma A1. Let $\zeta$ be a projector and $\operatorname{Rank} \zeta>s$. Then either $\zeta \in \mathscr{J}_{0}$ or it can be represented in the form

$$
\zeta=\tilde{\zeta}+\Delta
$$

where $\tilde{\zeta}$ and $\Delta$ are projectors, $\tilde{\zeta}$ is orthogonal to $\mathscr{J}_{0}$ and $\operatorname{Rank} \tilde{\zeta}>s$, and $\Delta \in \mathscr{J}_{0}$.

Proof. Lemma A1 is trivial if $\zeta$ is orthogonal to $\mathscr{J}_{0}$. Let now $\Delta \in \mathscr{J}_{0}$ be a projector with Rank $\Delta=s$ and $\zeta \Delta \neq 0$. Consider then a self-adjoined operator $\varphi:=\zeta \Delta \zeta$. Obviously $\operatorname{Rank} \varphi=s$, and hence for some real $c>0$ the operator $c \varphi$ is a projector and $c \varphi \in \mathscr{J}$. On the other hand $c \varphi$ is projecting on the same subspace that $\zeta$ (in this case we say $c \varphi \subset \zeta$ ). This implies that $\zeta_{1}:=\zeta-c \varphi$ is again a projector and obviously $\operatorname{Rank} \zeta_{1}=\operatorname{Rank} \zeta-s$. If $\zeta_{1}$ is not orthogonal to $\mathscr{J}_{0}$, we apply the same procedure to $\zeta_{1}$ and obtain $\zeta_{2}$ with $\operatorname{Rank} \zeta_{2}=\operatorname{Rank} \zeta-2 s$. We repeat this procedure up to the moment when we obtain some $\zeta_{r}$ with $\operatorname{Rank} \zeta_{r}=\operatorname{Rank} \zeta-r s$ and $\zeta_{r} \perp \mathscr{J}_{0}$ and $\zeta-\zeta_{r} \in \mathscr{J}_{0}$ by construction. Moreover, it is evident that $\zeta_{r} \subset \zeta$. We put now $\tilde{\zeta}=\zeta_{r}$ and $\Delta=Q-\tilde{\zeta}$.

Lemma A2. Suppose that $\mathscr{J}$ is irreducible. Then $\mathscr{J}=\mathscr{J}_{0}$.

Proof. Suppose that the set $\Gamma:=\{\tilde{\zeta}\}$ of those $\tilde{\zeta} \in \mathscr{J}$ which are orthogonal to $\mathscr{J}_{0}$ is not empty. It follows then from Lemma A1 that $\mathscr{J}=\mathscr{J}_{0} \oplus \Gamma$. But then the subspace of $\mathbb{R}^{m}$ which is equal to $\mathscr{J}_{0} \mathbb{R}^{m}$ is invariant with respect to the action of $\mathscr{J}$. This contradicts the irreducibility condition and shows that $\mathscr{J}_{0}=\mathscr{J}$.

Lemma A3. If $\mathscr{J}$ is irreducible and contains a projector of rank one then it contains all self-adjoined operators.

Proof. We know from Lemma A2 that the one-dimensional projectors belonging to $\mathscr{J}$ span $\mathscr{J}$. Now, it remains to show that all one-dimensional projectors belong to $\mathscr{J}$. Let $\Delta_{1}$ and $\Delta_{2}$ be two one-dimensional projectors which project onto lines generated by vectors $l_{1}$ and $l_{2}$ correspondingly and suppose that these vectors are not orthogonal, $\left(l_{1}, l_{2}\right) \neq 0$. Then $\mathscr{J}$ contains all the projectors which project onto lines belonging to a two-dimensional subspace generated by $l_{1}, l_{2}$.

Indeed, consider an operator

$$
\eta=\left(\Delta_{1}-\Delta_{2} \Delta_{1} \Delta_{2}\right) \Delta_{2}\left(\Delta_{1}-\Delta_{2} \Delta_{1} \Delta_{2}\right) .
$$

Evidently $\eta$ is self-adjoined, $\operatorname{Rank} \eta=\operatorname{Rank} \Delta_{2}=1$ and $\eta$ is orthogonal to $\Delta_{2}$ : $\eta \Delta_{2}=0$. The three operators $\eta, \Delta_{1}, \Delta_{2}$ form a basis in the 3-dimensional space of self-adjoined operators in the subspace generated by vectors $l_{1}, l_{2}$ and in particular contains all projectors mentioned above.

To finish the proof of our lemma consider now a subset $H \subset \mathbb{R}^{m}$ such that for every vector $x \in H$ there exists a projector $\Delta_{x} \in \mathscr{J}$, which projects onto the line, containing $x$. We have to show that $H=\mathbb{R}^{m}$. Suppose that $H \neq \mathbb{R}^{m}$. Then $H$ is not a linear space (otherwise this space would be invariant with respect to $\mathscr{J}$ ). Among all linear subspaces of $H$ pick one with a maximal dimension. Let it be $H_{0}, H_{0} \subset H$. As far as $H_{0} \neq H$, there exists a vector $l \in H \backslash H_{0}$ such that $l$ is not orthogonal to $H_{0}$ (here we use again the irreducibility condition). Now if $x \in H_{0}$ 
and $(x, l) \neq 0$ then as we have shown above $a x+b l \in H$. But $H$ is (evidently) a closed subset of $\mathbb{R}^{m}$ and therefore all the vectors of the form $a x+b l$, for every $x \in H_{0}$ and arbitrary real numbers $a, b$ belong to $L$. Thus the hypothesis that $H_{0}$ is a subspace of maximal dimension is not true and hence $H=\mathbb{R}^{m}$.

We need the following result about generalised Jordan algebras.

Lemma A4. Suppose that $\mathscr{R}$ is irreducible GJA which contains a projector of rank one. Then $\mathscr{R}$ is a Jordan algebra of all symmetric matrices.

Proof. It is clear that $\mathscr{R}$ is a JA if and only if $I \in \mathscr{R}$. We shall show that this relation holds. Then the fact that $\mathscr{R}$ contains all symmetric matrices is the statement of Lemma A3.

Consider the set of all projectors of rank one belonging to $\mathscr{R}$ and let $H$ be the subspace of $\mathbb{R}^{m}$ spanned by the vectors belonging to the ranges of these projectors. First of all, we establish that the dimension of $H$ is equal to $m$. Suppose that, on the contrary, $\operatorname{dim} H=s$ and $s<m$. Then pick a projector $\Delta$ with eigenvector $x$ such that there exists an operator $Q \in \mathscr{R}$ such that $Q x \notin H$ (we use here the irreducibility of $\mathscr{R})$. We put then $\eta:=Q \Delta Q \in \mathscr{R}$. Consider the spectral representation of $Q$

$$
Q=\sum_{i=1}^{k} \lambda_{i} u_{i}
$$

where $\lambda_{l}$ 's are different eigenvalues of $Q$ and $u_{i}$ are corresponding orthogonal projectors. We choose one of the projectors, say $u_{r}$, so that $u_{r} x \notin H$. Then

$$
\left(\eta u_{r} x, u_{r} x\right)=\left(\Delta Q u_{r} x, Q u_{r} x\right)=\lambda_{r}^{2}\left(\Delta u_{r} x, u_{r} x\right) \neq 0 .
$$

The fact that our scalar product is not equal to zero is a consequence of the choice of $u_{r}$, namely, $u_{r} x \neq 0$ and therefore not orthogonal to $x$. Thus we have that $\eta \neq 0$. On the other hand Rank $\eta=1$, and hence $\eta$ is proportional to a projector of rank one which belongs to $\mathscr{R}$. This latter projector by construction projects onto a line which does not belong to $H$. This contradicts the definition of $H$ unless $H=\mathbb{R}^{m}$.

We can now choose $m$ projectors $\Delta_{1}, \ldots, \Delta_{m}$ so that the eigenvectors of these projectors corresponding to the eigenvalue 1 form a basis in $\mathbb{R}^{m}$. Consider the operator $h:=\sum_{j=1}^{m} \Delta_{j} . h$ is a strictly positive operator and $h \in \mathscr{R}$. Applying to $h$ the same procedure that was used in Lemma 2.4 , we easily obtain that $I \in \mathscr{R}$.

Acknowledgements. I am grateful to G.A. Margulis for many useful discussions. The encouraging attitude to the work and generous hospitality and support of W. Kirsch and S. Albeverio were extremely helpful and important for me.

\section{References}

[A] Anderson, P.: Absence of diffusion in certain random lattices. Phys. Rev. 109, 1492 (1958)

[BL] Bougerol, P., Lacroix, J.: Products of Random Matrices with Applications to Schrödinger Operators. Boston, Basel, Stuttgart: Birkhäuser, 1985

[BK] Braun, H., Koecher, M.: Jordan-Algebren. Berlin, Heidelberg, New York: Springer, 1966, p. 352 
[CKM] Carmona, R., Klein, A., Martinelli, F.: Anderson localization for Bernoulli and other singular potentials. Commun. Math. Phys. 108, 41-66 (1987)

[CL] Carmona, R., Lacroix, J.: Spectral Theory of Random Schrödinger Operators. Boston: Birkhäuser, 1990

[FS] Fröhlich, J., Spencer, T.: Absence of diffusion in the Anderson tight binding model. Commun. Math. Phys. 88, 151-184 (1983)

[FMSS] Fröhlich, J., Martinelli, F., Scoppola, E., Spencer, T.: Constructive proof of localization in the Anderson tight binding model. Commun. Math. Phys. 101, 21-46 (1985)

[GMo] Goldsheid, I.Ya., Molchanov, S.A.: On the Mott problem. Dokl. Akad. Nauk. SSSR 230, 761-764 (1975)

[GMo] Goldsheid, I.Ya., Molchanov, S.A., Pastur, L.A.: One-dimensional random Schrödinger operator has a pure point spectrum. Func. Anal. Appl. 11, no. 1, 1-10 (1977)

[G] Goldsheid, I.Ya.: The structure of the spectrum of the Schrödinger random difference operator. Dokl. Akad. Nauk. SSSR 255, no. 2, 273-277 (1980)= Soviet Math. Dokl. 22, no. 2, 670-676 (1980)

[GM1] Goldsheid, I.Ya., Margulis, G.A.: Conditions of simplicity of the spectrum of Lyapunov indices. Dokl. Akad. Nauk SSSR 293, 297-301 (1987)= Soviet Math. Dokl. 35, 309-313 (1987)

[GM2] Goldsheid, I.Ya., Margulis, G.A.: Lyapunov indices of products of random matrices. Uspekhi Mat. Nauk 44:5, 13-60 (1989)=Russ. Math. Surv. 44:5, 11-71 (1989)

[GM3] Goldsheid, I.Ya., Margulis, G.A.: Point spectrum of operators and Lyapunov exponents. The Ninth School on Operators Theory, Abstracts, Tchelyabinsk, 1986

[Ki] Kingman, J.F.S.: Subadditive Processes. In: Ecole d'Eté de Probabilités de de Saint-Flour V, Lecture Notes in Math. 539, 1976, pp. 167-233

[KLS] Klein, A., Lacroix, J., Speis, A.: Localization for the Anderson model on a strip for singular potentials. J. Funct. Anal. 94, 135-155 (1990)

[KS] Kotani, S., Simon, B.: Stochastic Schrödinger operators and Jacobi Matrices on the strip. Commun. Math. Phys. 119, 403-429 (1988)

[Lal] Lacroix, J.: Localization pour l'opérateur de Schrödinger aléatoire dans un ruban. Ann. Inst. H. Poincaré, ser. A40, 97-116 (1984)

[La2] Lacroix, J.: The Schrödinger operator on a strip. Probability Measures on Groups VII, Lecture notes in Math. 1064, 1984, pp. 280-297

[L1] Ledrappier, F.: Quelques propriétés des exposants caractéristiques. Ecole d'Eté de Probabilités XIII Saint-Flour 1982. Springer Lect. Notes in Math. 1097, 1984

[L2] Ledrappier, F.: Positivity of the exponent for stationary sequences of matrices. Lecture notes in Mathematics 1186, Berlin, Heidelberg, New York: Springer, 1985, pp. 56-73

[O] Oseledets, V.I.: A multiplicative ergodic theorem, Characteristic Lyapunov exponents of dynamical systems. Trudy Moskov. Mat. Obshch. 19, 179-210 (1968)= Trans. Moscow Math. Soc. 19, 197-231 (1968)

[OV] Onishchik, A.L. and Vinberg, E.B.: Lie Groups and Algebraic Groups. Berlin, Heidelberg, New York: Springer, 1990

[R] Ruelle, D.: Characteristic exponents and invariant manifolds in Hilbert space. Ann. Math., Ser. 2, 115:2, 243-290 (1982) 
